\title{
Aa. Vv., L'Orthographe: du manuscrit médiéval à la linguistique moderne
}

\section{Gianni Mombello}

\section{Q OpenEdition}

10 Journals

\section{Édition électronique}

URL : http://journals.openedition.org/studifrancesi/35717

DOI : 10.4000/studifrancesi.35717

ISSN : 2421-5856

Éditeur

Rosenberg \& Sellier

\section{Édition imprimée}

Date de publication : 1 juillet 2005

Pagination : 132-133

ISSN : 0039-2944

\section{Référence électronique}

Gianni Mombello, « Aa. Vv., L'Orthographe: du manuscrit médiéval à la linguistique moderne », Studi

Francesi [En ligne], 145 (XLIX | I) | 2005, mis en ligne le 30 novembre 2015, consulté le 20 avril 2021.

URL : http://journals.openedition.org/studifrancesi/35717 ; DOI : https://doi.org/10.4000/

studifrancesi.35717

Ce document a été généré automatiquement le 20 avril 2021.

\section{(c)}

Studi Francesi è distribuita con Licenza Creative Commons Attribuzione - Non commerciale - Non opere derivate 4.0 Internazionale. 


\title{
Aa. Vv., L'Orthographe: du manuscrit médiéval à la linguistique moderne
}

\author{
Gianni Mombello
}

\section{RÉFÉRENCE}

YVONNE CAZAL, GABRIELLA PARUSSA, CINZIA PIGNATELLI et RICHARD TRACHSLER, L'Orthographe: du manuscrit médiéval à la linguistique moderne, «Médiévales», 45, 2003, pp. 99-116.

1 Ce bref compte rendu d'une recherche d'envergure, mais qui est encore à ses débuts, nous offre des données à retenir et surtout des perspectives que les anglophones définiraient «seminal». Elle est fondée sur 900.000 mots tirés d'une dizaine de manuscrits (dont six signalés) d'auteurs et copistes actifs au cours de la première moitié du XVe siècle (Raoul Tainguy, Christine de Pizan et Rasse de Brunhamel). Elle semble aller dans une direction (trouver les critères pour identifier une main), mais elle aboutit à une autre (étude des tendances orthographiques du $\mathrm{XV}^{\mathrm{e}}$ siècle).

2 Une fois écartées les graphies «indifférentes» (e. g., 'chevalier', 'chevallier'), les chercheurs se penchent sur les graphies «privilégiées», dans l'espoir de saisir le «tic» du copiste qui permette son identification. Ce «tic», hélas, n'est pas facile à repérer.

3 Une fois établi qu'un copiste suit un système privilégié, quoique non uniforme, les auteurs de cet article s'interrogent aussi sur la possibilité de l'existence d'un système supra-individuel, en procédant par étapes. Par exemple, ils examinent la présence de consonnes etymologiques, ou prétendues telles, en fouillant dans les failles des différents systèmes adoptés. Ils en dégagent des observations fort intéressantes sur l'apparition du ' $h$ ' à l'initiale, du 'p' dans 'escripre', sur la présence de 'bz/bs' dans des mots composés par le résultat de 'sub' plus un deuxième élément qui a une existence propre (ex: 'soubzrire'), mais jamais dans 'soubdain', 'soubtil', etc. Si pour le 'p' d'escripre' et le ' $n$ ' de 'prins' on signale justement l'influence de l'analogie avec les formes latines ou franciennes ayant ces lettres (ou plutôt, ces phonèmes, détail fondamental) ('scriptu', 'prendre'), il me semble qu'on a prêté moins d'attention à un 
autre phénomène connu comme «manie des hampes» et le 'p' en a une justement. En outre, en parlant des lettres quiescentes, pourquoi ne pas mettre l'accent sur la nécessité absolue de distinguer les homophones et les mots tendant à l'homophonie? En effet, comment représenter 's(e)i' >'se': 'sain', 'saint', 'sein', 'ceint', 'seing'...? Vivement les lettres étymologiques (ou prétendues telles)!

4 A propos de l'apparition du ' $n$ ' dit parasite dans 'prins' et surtout du verbe et substantif 'souvenir', qui ne semble avoir jamais eu de 'b', «seule exception à la règle de la préfixation» (p. 111), j'ai une suggestion apparemment saugrenue à faire; c'est parce que ces deux lexèmes (et surtout le dernier) pourraient avoir pris un autre chemin sur le plan phonétique.

5 En examinant un incunable de la fin du XVe siecle (Les Ditz des sages hommes de Guillaume Tardif), je suis tombé sur 'sonvenir'. Bien entendu, ' $u$ ' et ' $n$ ' sont interchangeables, mais il y a d'autres attestations dans ce même texte: 'gonverneur', 'sonstenir', 'sonvenance', etc. D'ailleurs, 'mont' pour 'mout'>'molt', 'monton' pour 'mouton' sont largement attestés (ce dernier étant différemment expliqué). Le TL signale la forme 'temonte'> 'tumultu' garanti par la rime ('remonte', 'monte') dans l'Escoufle (vv. 4008 et 4095) et le FEW le dialectal 'monche' pour 'mouche' (pour ne pas parler de 'convoiter'>*cupidietare qui aurait été calqué sur 'covenir', 'convenir', selon Fouché, pp. 800-01, de 'covent', 'couvent', 'convent', ibid.). Puisqu'ils y sont, pourquoi nos quatre mousquetaires ne vérifieraient-ils pas, chez des scribes qui distingueraient avec une très haute fréquence ' $u$ ' de ' $n$ ', si cette consonne nasale fait son apparition dans des mots (ou dans des mots proches?) où il y a déjà un autre élément nasal? Cela serait un indice précieux pour prouver l'extension (progressive ou régressive) de la nasalisation, un phénomène qui ne semble pas encore avoir été examiné. Je sais bien que cela est autant, sinon plus difficile que de prouver, avec précision, la date à laquelle 'pouoir' est devenu 'po(u)voir', mais on peut toujours tenter l'aventure, maintenant qu'on possède des moyens informatiques que le regretté Omer Jodogne ne possédait pas, et surtout parce que ' $u$ ' et 'n'ont un tracé inverse, tandis que ' $u$ ' et 'v' étaient indistincts jusqu'à l'adoption généralisée (fort tardive) des lettres ramistes.

6 Les observations se rapportant à la morphologie des verbes sont tout aussi intéressantes, mais il faut se borner, autrement on déborde des limites d'une fiche signalétique. Lorsque l'alternance du radical oppose le présent de l'indicatif à celui du subjonctif, l'alternance des désinences en '-iez', '-ez', pourrait devenir superflue comme dans le verbe 'savoir', et pourtant le français l'a conservée, puisque ce cas est rare. Dans l'établissement du système de la conjugaison française, le maître d'œuvre reste l'analogie.

7 Juste une dernier mot. Nos quatre explorateurs sont des pionniers hardis. Il faut leur souhaiter bien de la chance parce qu'ils ont devant eux une forêt «selvaggia e aspra e forte». En effet après les nombreuses tentatives de réforme du XVI siècle, qui ont abouti à un échec escompté, puisqu'elles prétendaient fonder l'orthographe sur la prononciation, qui était, alors et longtemps encore par la suite, fort variable et surtout parce que, en ce qui concerne le français, mais pas seulement le français, l'orthographe n'est pas un code substitutif de la phonétique, les imprimeurs humanistes lui ont imposé un carcan étymologisant assez sensé mais fort différencié d'un atelier à l'autre. Le Classicisme, qui a prétendu tout régler, a flairé l'embûche et s'est bien gardé d'intervenir, dans ce domaine, sinon épisodiquement. La normalisation, si on peut l'appeler ainsi, a été l'affaire du temps. Encore au milieu du XVII ${ }^{e}$ siècle, Vaugelas se 
plaignait, dans une lettre que j'ai publiée, qu'un copiste téméraire avait osé modifier «son» («mon» dans le texte) orthographe. Un crime de lèse-majesté, pour un académicien; mais autant dire que chacun avait la sienne, qu'il défendait comme la meilleure possible (évidemment à un très haut niveau). En ce qui concerne l'orthographe «commune», celle des correspondances, par exemple, et surtout celle des lettres écrites par des femmes, même les plus lettrées, comme Madame de La Fayette, la faible connaissance ou l'ignorance du latin se fait sentir et on risque très souvent l'illisibilité.

8 Si l'orthographe a été longtemps un fait assez, sinon exclusivement, personnel, ou d'atelier, on a la chance d'identifier, tôt ou tard, des constantes et partant des mains; mais gare à se fier seulement à l'orthographe; avant et surtout après l'imprimerie, le ductus a son mot à dire. 